\title{
Distribuição das características clínicas do fenótipo gengival em pacientes saudáveis
}

\author{
Distribution of clinical characteristics of gingival phenotype in healthy patients
}

\author{
Samuel Batista BORGES ${ }^{a *}$ (1), Lidya Nara Marques de ARAÚJOa (1), \\ Bruno César de Vasconcelos GURGEL ${ }^{a}$ (D) \\ aUFRN - Universidade Federal do Rio Grande do Norte, Natal, RN, Brasil
}

Como citar: Borges SB, Araújo LNM, Gurgel BCV. Distribuição das características clínicas do fenótipo gengival em pacientes saudáveis. Rev Odontol UNESP. 2019;48:e20190011. https://doi.org/10.1590/1807-2577.01119

\begin{abstract}
Resumo
Introdução: A determinação do fenótipo gengival possibilita compreender melhor as variações e diferenças clínicas intra e interindividuais dos tecidos periodontais saudáveis. Objetivo: 0 objetivo deste estudo foi determinar os perfis do fenótipo gengival e compará-los em diferentes regiões da cavidade oral, levando em consideração parâmetros clínicos relacionados aos tecidos moles circunjacentes nos dentes anteriores superiores e inferiores. Material e método: Noventa e um pacientes com saúde periodontal foram examinados e os parâmetros clínicos profundidade de sondagem (PS), largura de mucosa ceratinizada (MC), espessura gengival em ambos incisivos centrais superiores (EG) e fenótipo gengival (FG) foram coletados nos dentes anteriores superiores e inferiores. Os dados foram analisados estatisticamente pelos testes $t$-Student pareado $\mathrm{e}$ independente, Correlação de Pearson e Qui-quadrado, com um nível de significância de 5\%. Resultado: 0 fenótipo gengival espesso predominou nos dentes anteriores superiores $(60,5 \%)$, enquanto o fenótipo fino foi mais prevalente nos dentes anteriores inferiores $(84,6 \%)$. Diferenças estatisticamente significativas foram encontradas para os parâmetros largura de mucosa ceratinizada e espessura gengival em relação aos fenótipos gengivais superiores e inferiores $(p<0,05)$. Não houve diferença estatística entre profundidade de sondagem e o fenótipo gengival nos dentes superiores e inferiores. Conclusão: Concluiu-se que o fenótipo gengival espesso é mais frequente nos dentes anteriores superiores e que o fenótipo fino é mais comum nos dentes anteriores inferiores, sendo mais frequente em indivíduos do sexo masculino. Os parâmetros largura de mucosa ceratinizada e espessura gengival mostraram-se adequados à determinação do fenótipo gengival.
\end{abstract}

Descritores: Periodontia; gengiva; fenótipo; diagnóstico.

\begin{abstract}
Introduction: Gingival phenotype determination enables a better understanding of intra and interindividual clinical variations in healthy periodontal tissues. Objective: The aim of this study was to determine gingival phenotype profiles and compare these in different regions of the oral cavity, considering clinical parameters related to the surrounding soft tissue in the upper and lower front teeth. Material and method: Ninety-one periodontally-healthy patients were examined and clinical parameters such as probing depth (PD), keratinized tissue width (KW), gingival thickness at both maxillary central incisors (GT) and gingival phenotype (GP) were determined for the upper and lower front teeth. Data were statistically analyzed by paired and independent $t$-Student tests, Pearson Correlation and the Chi-square test, using a significance level of 5\%. Result: The thick gingival phenotype predominated in the upper anterior teeth (60.5\%), while the thin phenotype was more prevalent in the lower anterior teeth (84.6\%). Statistically significant differences were found for keratinized tissue and gingival thickness parameters, in relation to the upper and lower gingival phenotypes $(\mathrm{p}<0.05)$. There were no statistical significances for probing depth and gingival phenotype in the upper and lower teeth. Conclusion: It was concluded that the thick phenotype is more frequent in the upper anterior teeth and that the thin phenotype is more common in the lower anterior teeth, and more frequent in males. The keratinized tissue and gingival thickness parameters proved to be suitable for the determination of gingival phenotype.
\end{abstract}

Descriptors: Periodontics; gingiva; phenotype; diagnosis. 


\section{INTRODUÇÃO}

O fenótipo gengival, segundo a nova classificação das doenças e condições periodontais e peri-implantares da Academia Americana de Periodontia e da Federação Europeia de Periodontia ${ }^{1,2}$ (2017), tem sido considerado como um aspecto da gengiva que indica uma dimensão passível de ser mudada ao longo do tempo - dependente de uma combinação multifatorial de características genéticas, fatores ambientais e intervenção clínica - e que pode ser específica do local.

Os parâmetros clínicos utilizados para avaliar o fenótipo gengival têm sido evidenciados em especialidades da Odontologia, como Ortodontia, Implantodontia, Prótese Dentária e Dentística Estética e Restauradora ${ }^{3,4}$, e isso se deve ao fato de, normalmente, os tecidos periodontais saudáveis apresentarem variações clínicas intra e interindividuais ${ }^{5,6}$. A identificação prévia do fenótipo gengival é importante para definir fatores relacionados ao arcabouço periodontal, uma vez que estes podem ter um efeito significativo nos procedimentos terapêuticos restauradores e reabilitadores ${ }^{3,4,7}$.

A fim de minimizar possíveis danos secundários às terapias odontológicas e procedimentos cirúrgicos mucogengivais, a determinação do fenótipo gengival tem viabilizado a observação de diferentes tipos de respostas teciduais a processos inflamatórios decorrentes de intervenções estéticas e reabilitadoras 7,8 , garantindo um melhor prognóstico para os tratamentos empregados, uma vez que planejamentos prévios deveriam levar em conta esse parâmetro periodontal.

De acordo com a nova classificação das doenças e condições periodontais e peri-implantares da Academia Americana de Periodontia e da Federação Europeia de Periodontia7,8, os principais parâmetros clínicos para identificação do fenótipo gengival, de forma padronizada, baseiam-se principalmente na determinação do volume gengival tridimensional, a partir da avaliação da largura da faixa de mucosa ceratinizada (MC) e da espessura gengival (EG), que pode ser aferida através do teste de transparência à sondagem ${ }^{8,9}$ ou pela sondagem transgengival ${ }^{10}$.

Estudos de autores, como De Rouck et al. ${ }^{8}$ e Kan et al. ${ }^{9}$, demonstraram a eficácia clínica desses testes ao observar a reprodutibilidade deles em indivíduos com diferentes espessuras gengivais e estruturas ósseas periodontais. No entanto, reproduzindo os experimentos anteriores, Anand et al. ${ }^{10}$ e Bhat, Shetty ${ }^{11}$ descreveram a falta de uma única relação significativa entre os parâmetros clínicos, quando analisados separadamente para a determinação do fenótipo gengival em relação às combinações morfométricas dentárias em regiões distintas na cavidade oral.

Sob a perspectiva da pesquisa e das práticas clínicas odontológicas cotidianas, com ênfase nas características periodontais individuais dos pacientes e em um bom planejamento terapêutico, se faz necessário o estudo do fenótipo gengival para um correto diagnóstico. Dessa maneira, o objetivo deste estudo foi determinar os perfis do fenótipo gengival e compará-los em diferentes regiões da cavidade oral, levando em consideração parâmetros clínicos relacionados aos tecidos moles circunjacentes nos dentes anteriores superiores e inferiores.

\section{MATERIAL E MÉTODO}

Este estudo observacional do tipo transversal foi aprovado pelo Comitê de Ética em Pesquisa com Seres Humanos da Universidade Federal do Rio Grande do Norte (UFRN) sob o parecer número 909.875/2014. A inclusão dos indivíduos no estudo se deu após assinatura de Termo de Consentimento Livre e Esclarecido (TCLE).

A amostra do estudo foi não probabilística e a amostragem consistiu de estudantes de Odontologia da Universidade Federal do Rio Grande do Norte (UFRN), acima dos 18 anos de idade, com estado de saúde clínica em um periodonto íntegro ${ }^{12}$ (sem perda de inserção, profundidade de sondagem de até $3 \mathrm{~mm}$, sangramento à sondagem em menos de $10 \%$ dos sítios 
e sem perda óssea radiográfica), que apresentaram todos os dentes anteriores superiores e inferiores, e aceitaram o convite para participar desta pesquisa. Não foram incluídos na pesquisa indivíduos com restaurações ou próteses na coroa nos dentes anteriores e/ou com aparelhos ortodônticos; fumantes; grávidas ou lactentes; sujeitos submetidos à terapêutica medicamentosa com efeitos conhecidos sobre os tecidos periodontais moles, e/ou voluntários com histórico de tratamento da periodontite.

Para o cálculo amostral, foi considerada a proporção de fenótipos espesso e fino baseada no estudo de De Rouck et al. ${ }^{8}$, que obteve uma relação de dois terços de gengiva espessa ao teste de transparência à sondagem (TS). 0 erro admitido foi de 10\%, nível de significância de 5\% e poder do teste de $90 \%$, totalizando 87 indivíduos. A amostra final foi constituída de 91 indivíduos.

\section{Coleta de Dados}

A coleta de dados foi realizada no período compreendido entre janeiro e junho de 2015, nas clínicas do Departamento de Odontologia da Universidade Federal do Rio Grande do Norte (UFRN), por um único examinador, que foi calibrado para os parâmetros MC $(\mathrm{ICC}=0,634 ; p<0,001)$ e fenótipo gengival (Kappa=0,667; $p<0,05)$. Todos os pacientes foram submetidos a anamnese e exame clínico, conforme descrito a seguir, adaptados das metodologias de De Rouck et al. ${ }^{8}$, Anand et al.10 e Cuny-Houchmand et al.13. A análise e a descrição do fenótipo gengival (FG) foram realizadas a partir do teste de transparência à sondagem (TS) nos incisivos centrais superiores e inferiores, com sonda periodontal da Carolina do Norte UNC-15 (Hu-Friedy ${ }^{\circledR}$, Chicago, IL, EUA).

Os elementos dentários cujas transparências foram positivas através da margem gengival determinaram a classificação do FG em fino. Em casos contrários, o fenótipo era classificado como espesso. Para os padrões de determinação da profundidade de sondagem (PS), foi realizada sondagem em três sítios de cada um dos seis elementos anteriores superiores e inferiores, com registro estimado até os $0,5 \mathrm{~mm}$ mais próximos ${ }^{8}$, sendo então calculada a média aritmética para cada dente e para os sextantes anteriores superior e inferior.

Para medir a faixa de mucosa ceratinizada (MC), tomou-se a medida do ponto mais coronal da margem gengival de cada elemento dentário até a junção mucogengival, com auxílio da sonda periodontal. 0 teste de espessura gengival consistiu na inserção de um espaçador digital endodôntico cônico com cursor de silicone (Maillefer ${ }^{\mathrm{T}}$ Dentsply $^{\circledR}$, Tulsa, OK, EUA) na região média da gengiva inserida de cada um dos incisivos centrais superiores e inferiores, previamente anestesiada com solução anestésica tópica. Após esse procedimento, foram feitas mensurações com o uso de paquímetro digital (Mitutoyo ${ }^{\circledR}$, Kawasaki, KW, Japão). A exposição gengival ao sorrir foi medida também com sonda periodontal milimetrada, mensurando a distância de um ponto central da porção mais coronal da gengiva marginal dos incisivos superiores à borda inferior do lábio superior.

\section{Análise dos Dados}

O software Statistic Data Analysis - STATA $13.0^{\circledR}$ (STATACorp, LLC, Texas, EUA) foi utilizado para armazenamento e análise de dados. Inicialmente, uma análise descritiva dos dados foi realizada para uma avaliação da distribuição da normalidade dos dados quantitativos (PS, MC, EG), utilizando os dados do desvio padrão, da assimetria e da curtose. Testes paramétricos foram utilizados visto que os dados apresentaram distribuição normal.

Para avaliar as diferenças significativas dentro dos grupos de dentes, foi aplicado o teste t emparelhado de Student. Para a comparação intergrupo (dentes superiores versus inferiores), o teste $t$ de Student para amostras independentes foi utilizado. Para análise do 
dado categórico fenótipo gengival e a sua associação com outras variáveis independentes categóricas (sexo, raça e exposição gengival ao sorrir), foi utilizado o teste Qui-quadrado.

Com finalidade de aperfeiçoar a análise estatística para esse teste, as variáveis categóricas raça e exposição gengival ao sorrir foram recategorizadas. Raça foi recategorizada em branca e negra/parda, e exposição gengival ao sorrir foi recategorizada em $<2 \mathrm{~mm}$ e $>2 \mathrm{~mm}$. Para todos os testes, o indivíduo foi considerado a unidade de análise e foi pré-estabelecido um nível de significância de $5 \%(\alpha=0,05)$. Por fim, foi usado o Teste de Correlação de Pearson entre as médias (em milímetros) de faixas de MC de diferentes grupos de elementos superiores e inferiores aferidas, bem como de fenótipo gengival superior e inferior com as médias de PS de incisivos centrais superiores e inferiores, respectivamente.

\section{RESULTADO}

Entre os 91 indivíduos, a maior frequência foi do sexo masculino (51,6\%), com idade média de 22,73 anos $( \pm 1,49)$ e variação entre 20 e 27 anos. Quanto a raça ou cor, predominou a branca $(n=54 ; 59,3 \%)$, seguida pelas raças parda $(n=31 ; 34,1 \%)$ e negra $(n=6 ; 6,6 \%)$. A exposição gengival ao sorrir predominou entre a faixa de 2 a $4 \mathrm{~mm}(\mathrm{n}=46 ; 50,5 \%)$, seguida pela faixa inferior a $2 \mathrm{~mm}(\mathrm{n}=40,44 \%)$ e pela faixa superior a $4 \mathrm{~mm}(\mathrm{n}=5 ; 5,5 \%)$. Quanto ao fenótipo gengival, o tipo espesso predominou na região superior $(n=55 ; 60,5 \%)$, enquanto que o fenótipo fino foi mais prevalente na região inferior ( $\mathrm{n}=77 ; 84,6 \%)$.

A associação entre sexo e fenótipo gengival superior revelou que indivíduos do sexo masculino exibiram fenótipo gengival mais espesso $(n=38 ; 80,85 \%)$. 0 mesmo ocorreu ao ser analisada a associação entre exposição gengival ao sorrir e fenótipo gengival superior, em que foi visto que indivíduos com fenótipo espesso possuíram exposição maior que $2 \mathrm{~mm}$ (Tabela 1).

Tabela 1. Associação do fenótipo gengival superior com variáveis independentes e associação de exposição gengival ao sorrir com sexo. Teste Qui-quadrado. Natal, RN, 2019

\begin{tabular}{|c|c|c|c|c|}
\hline \multirow{2}{*}{ Parâmetros } & \multicolumn{2}{|c|}{ Fenótipo Gengival Superior } & \multirow{2}{*}{$\begin{array}{l}\text { Total } \\
\text { n (\%) }\end{array}$} & \multirow{2}{*}{$p$-valor } \\
\hline & Fino n (\%) & Espesso n (\%) & & \\
\hline \multicolumn{5}{|l|}{ Sexo } \\
\hline Masculino & $9(19,15)$ & $38(80,85)$ & $47(100)$ & \multirow{2}{*}{$<0,001$} \\
\hline Feminino & $27(61,4)$ & $17(38,6)$ & $44(100)$ & \\
\hline \multicolumn{5}{|l|}{ Raça } \\
\hline Branca & $23(42,6)$ & $31(57,4)$ & $54(100)$ & \multirow{2}{*}{0,62} \\
\hline Negra/Parda & $14(27,5)$ & $24(64,9)$ & $37(100)$ & \\
\hline \multicolumn{5}{|c|}{ Exposição Gengival ao Sorrir } \\
\hline$<2 \mathrm{~mm}$ & $22(55)$ & $18(45)$ & $40(100)$ & \multirow{2}{*}{0,014} \\
\hline \multirow[t]{3}{*}{$>2 \mathrm{~mm}$} & $11(12,1)$ & $37(72,5)$ & $51(100)$ & \\
\hline & \multicolumn{2}{|c|}{ Exposição Gengival ao Sorrir } & Total & \multirow{2}{*}{$p$-valor } \\
\hline & $<2 \mathrm{~mm} \mathrm{n}(\%)$ & $>2 \mathrm{~mm} \mathrm{n}(\%)$ & n (\%) & \\
\hline \multicolumn{5}{|l|}{ Sexo } \\
\hline Masculino & $18(38,3)$ & $29(61,7)$ & $47(100)$ & \multirow{2}{*}{0,361} \\
\hline Feminino & $22(50)$ & $22(50)$ & $44(100)$ & \\
\hline
\end{tabular}

Fonte: Dados da Pesquisa.

A análise emparelhada das médias das faixas de mucosa ceratinizada dos grupos de dentes avaliados mostrou que houve diferença entre dentes superiores e inferiores $(p<0,001)$, em que a média da mucosa dos dentes anteriores superiores possui quase o dobro do valor médio da mucosa dos elementos equivalentes no arco inferior (Tabela 2). A média da espessura gengival também foi maior nos incisivos centrais superiores do que nos incisivos centrais inferiores $(p<0,001)$ (Tabela 2). 
Tabela 2. Diferença entre médias (em milímetros) e desvio padrão (DP) das faixas de mucosa ceratinizada de caninos e incisivos superiores e inferiores, e diferença entre médias de espessura gengival de incisivos centrais superiores e inferiores. Teste $t$-Student pareado. Natal, RN, 2019

\begin{tabular}{|c|c|c|c|c|c|c|}
\hline \multirow{2}{*}{ Parâmetros } & \multirow{2}{*}{$\mathbf{n}$} & \multirow{2}{*}{ Média (mm) } & \multirow{2}{*}{ DP } & \multicolumn{2}{|c|}{ IC (95\%) } & \multirow{2}{*}{$p$-valor } \\
\hline & & & & LI & LS & \\
\hline \multicolumn{7}{|c|}{ Mucosa Ceratinizada } \\
\hline Dentes Superiores & 91 & 6,23 & $\pm 1,86$ & 5,87 & 6,61 & \multirow{2}{*}{$<0,001$} \\
\hline Dentes Inferiores & 91 & 3,17 & $\pm 1,10$ & 2,96 & 3,39 & \\
\hline Incisivos Superiores & 91 & 6,67 & $\pm 1,92$ & 6,25 & 7,09 & \multirow{2}{*}{$<0,001$} \\
\hline Caninos Superiores & 91 & 5,79 & $\pm 1,85$ & 5,37 & 6,16 & \\
\hline Incisivos Inferiores & 91 & 3,40 & $\pm 1,19$ & 3,17 & 3,63 & \multirow{2}{*}{$<0,001$} \\
\hline Caninos Inferiores & 91 & 2,94 & $\pm 1,11$ & 2,72 & 3,15 & \\
\hline \multicolumn{7}{|c|}{ Espessura Gengival } \\
\hline Incisivos Centrais Superiores & 91 & 1,17 & $\pm 0,32$ & 1,06 & 1,24 & \multirow{2}{*}{$<0,001$} \\
\hline Incisivos Centrais Inferiores & 91 & 0,70 & $\pm 0,19$ & 0,66 & 0,74 & \\
\hline
\end{tabular}

DP (desvio padrão); IC (intervalo de confiança); LI (limite inferior); LS (limite superior). Fonte: Dados da Pesquisa.

Foi verificada diferença estatística na análise das médias de mucosa ceratinizada de caninos e incisivos tanto superiores $(p<0,001)$ quanto inferiores $(p<0,001)$. A faixa de mucosa mostrou-se maior tanto para os incisivos superiores quanto para os inferiores em relação aos caninos superiores e inferiores, respectivamente (Tabela 2).

Houve correlação positiva moderada, com significância estatística, entre MC dos incisivos centrais superiores e EG superior $(r=0,817 ; p<0,001)$, e entre EG dos incisivos inferiores e EG dos incisivos superiores $(r=0,32 ; p=0,02)$, bem como houve correlação positiva fraca, com significância estatística, para MC dos dentes superiores e MC dos dentes inferiores $(r=0,239$; $p=0,022)$, e entre MC incisivos centrais inferiores e EG dos incisivos inferiores $(r=0,234 ; p=0,026)$. Nesses casos, a proporcionalidade das correlações ainda é mantida (Tabela 3).

Tabela 3. Correlação das médias (em milímetros) de mucosa ceratinizada de diferentes grupos de dentes superiores e inferiores, e dos fenótipos gengivais superiores e inferiores com as médias da profundidade de sondagem dos incisivos centrais superiores e inferiores, respectivamente. Correlação de Pearson. Natal, RN, 2019

\begin{tabular}{|c|c|c|}
\hline & \multicolumn{2}{|c|}{ MCS } \\
\hline & $r$ & $p$-valor \\
\hline \multirow[t]{3}{*}{ MCI } & 0,239 & 0,022 \\
\hline & \multicolumn{2}{|c|}{ MCCS } \\
\hline & $r$ & $p$-valor \\
\hline \multirow[t]{3}{*}{ MCIS } & 0,942 & $<0,001$ \\
\hline & \multicolumn{2}{|c|}{ MCCI } \\
\hline & $r$ & $p$-valor \\
\hline \multirow[t]{3}{*}{ MCII } & 0,817 & $<0,001$ \\
\hline & \multicolumn{2}{|c|}{ EGI } \\
\hline & $r$ & $p$-valor \\
\hline \multirow{3}{*}{ EGS } & 0,32 & 0,002 \\
\hline & \multicolumn{2}{|c|}{ MCICS } \\
\hline & $r$ & $p$-valor \\
\hline \multirow{3}{*}{ EGS } & 0,597 & $<0,001$ \\
\hline & \multicolumn{2}{|c|}{ MCICI } \\
\hline & $r$ & $p$-valor \\
\hline EGI & 0,234 & 0,026 \\
\hline
\end{tabular}


Tabela 3. Continuação...

\begin{tabular}{cccc}
\hline & \multicolumn{2}{c}{ FGS } \\
\cline { 2 - 3 } & & $\boldsymbol{r}$ & $\boldsymbol{p}$-valor \\
\hline PSICS & 0,107 & 0,313 \\
\hline & & FGI \\
\cline { 2 - 3 } & $\boldsymbol{r}$ & $\boldsymbol{p}$-valor \\
\hline PSICI & 0,039 & 0,716 \\
\hline
\end{tabular}

$r$ (coeficiente de correlação de Pearson); MCS (mucosa ceratinizada de dentes superiores de canino a canino); MCI (mucosa ceratinizada de dentes inferiores de canino a canino); MCIS (mucosa ceratinizada incisivos superiores); MCII (mucosa ceratinizada incisivos inferiores); MCCS (mucosa ceratinizada dos caninos superiores); MCCI (mucosa ceratinizada dos caninos inferiores); EGS (espessura gengival superior); EGI (espessura gengival inferior); MCICS (mucosa ceratinizada dos incisivos e caninos superiores); MCICI (mucosa ceratinizada dos incisivos e caninos inferiores); PSICS (profundidade de sondagem de incisivos centrais superiores); PSICI (profundidade de sondagem de incisivos centrais inferiores); FGS (fenótipo gengival superior); FGI (fenótipo gengival inferior). Fonte: Dados da Pesquisa.

A análise do FG em relação à média das faixas de mucosa ceratinizada de incisivos superiores e inferiores apontou diferença estatística significativa, indicando que indivíduos com fenótipo gengival superior espesso possuem maior faixa de mucosa nos incisivos superiores do que os que possuem fenótipo gengival superior fino. 0 mesmo ocorreu para indivíduos com fenótipo gengival inferior espesso, embora maior quantidade de fenótipos gengivais inferiores tenha sido fina (Tabela 4).

Tabela 4. Diferenças entre médias (em milímetros) e desvios padrão (DP) das faixas de mucosa ceratinizada de incisivos superiores e inferiores, tipos de fenótipos gengivais superiores e inferiores, média de espessuras gengivais superiores e inferiores, e diferença entre médias de faixas de mucosa ceratinizada de dentes anteriores superiores e inferiores em relação ao sexo. Teste $t$-Student independente. Natal, RN, 2019

\begin{tabular}{|c|c|c|c|c|c|c|c|}
\hline & & \multirow{2}{*}{$\mathbf{n}$} & \multirow{2}{*}{$\begin{array}{l}\text { Média } \\
(\mathrm{mm})\end{array}$} & \multirow{2}{*}{ DP } & \multicolumn{2}{|c|}{ IC $(95 \%)$} & \multirow{2}{*}{$p$-valor } \\
\hline & & & & & LI & LS & \\
\hline & & \multicolumn{6}{|c|}{ MCIS } \\
\hline \multirow{2}{*}{ FGS } & Fino & 36 & 4,90 & $\pm 0,99$ & 4,56 & 5,24 & \multirow{2}{*}{$<0,001$} \\
\hline & Espesso & 55 & 7,83 & $\pm 1,43$ & 7,45 & 8,22 & \\
\hline & & \multicolumn{6}{|c|}{ EGS } \\
\hline \multirow{2}{*}{ FGS } & Fino & 36 & 0,88 & $\pm 0,27$ & 0,78 & 0,97 & \multirow{2}{*}{$<0,001$} \\
\hline & Espesso & 55 & 1,37 & $\pm 0,17$ & 1,32 & 1,41 & \\
\hline & & \multicolumn{6}{|c|}{ MCII } \\
\hline \multirow{2}{*}{ FGI } & Fino & 77 & 3,29 & $\pm 1,09$ & 3,04 & 3,54 & \multirow{2}{*}{0,036} \\
\hline & Espesso & 14 & 4,02 & $\pm 1,52$ & 3,14 & 4,89 & \\
\hline & & \multicolumn{6}{|c|}{ EGI } \\
\hline \multirow{2}{*}{ FGI } & Fino & 77 & 0,66 & $\pm 0,12$ & 0,63 & 0,69 & \multirow{2}{*}{0,016} \\
\hline & Espesso & 14 & 0,9 & $\pm 0,32$ & 0,71 & 1,08 & \\
\hline & & \multicolumn{6}{|c|}{ MCS } \\
\hline \multirow{2}{*}{ Sexo } & Masculino & 47 & 7,35 & $\pm 2,01$ & 6,75 & 7,93 & \multirow{2}{*}{$<0,001$} \\
\hline & Feminino & 44 & 5,96 & $\pm 1,54$ & 5,49 & 6,43 & \\
\hline & & \multicolumn{6}{|c|}{ MCI } \\
\hline \multirow{2}{*}{ Sexo } & Masculino & 47 & 3,43 & $\pm 1,26$ & 3,06 & 3,80 & \multirow{2}{*}{0,711} \\
\hline & Feminino & 44 & 3,37 & $\pm 1,12$ & 3,03 & 3,71 & \\
\hline
\end{tabular}

DP (desvio padrão); IC (intervalo de confiança); LI (limite inferior); LS (limite superior); FGS (fenótipo gengival superior); FGI (fenótipo gengival inferior); EGS (espessura gengival superior); EGI (espessura gengival inferior); MCS (mucosa ceratinizada de dentes superiores de canino a canino); MCI (mucosa ceratinizada de dentes inferiores de canino a canino); MCIS (mucosa ceratinizada incisivos superiores); MCII (mucosa ceratinizada incisivos inferiores). Fonte: Dados da Pesquisa.

Em relação à espessura gengival, pôde-se verificar que indivíduos com fenótipo gengival superior espesso possuem maior média (em milímetros) de espessura gengival superior do que 
aqueles cujo fenótipo é fino. No caso de indivíduos com fenótipo gengival inferior espesso, a média de espessura gengival inferior também foi maior. Quando foram analisadas as médias de mucosa dos dentes (canino a canino) superiores e inferiores em relação ao sexo, foi possível perceber que existe diferença estatística entre sexo masculino e feminino, sendo que homens apresentaram maior média de mucosa ceratinizada. Não houve diferença estatística para sexo e MC dos dentes (canino a canino) inferiores (Tabela 4).

\section{DISCUSSÃO}

Para a execução de tratamentos previsíveis e que garantam o sucesso das terapias de reabilitação, para o restabelecimento da função, melhora estética e manutenção da saúde periodontal, vários autores têm se engajado no estudo do fenótipo gengival ${ }^{8-11,13}$. Como resultado, várias alternativas diagnósticas têm sido sugeridas na literatura, embora nenhuma delas seja precisa, devido à dificuldade em medir e quantificar todos os parâmetros que determinam cada tipo de fenótipo.

Mais notavelmente, tem se destacado o método de classificação proposto nos estudos de De Rouck et al. ${ }^{8}$ e Kan et al. ${ }^{9}$, em que o fenótipo gengival é determinado pelo teste de transparência à sondagem (TS) em incisivos centrais superiores. Esses autores encontraram uma prevalência de $63 \%$ e $50 \%$ para fenótipos gengivais com gengiva espessa, respectivamente. 0 presente estudo encontrou uma prevalência de aproximadamente $60 \%$ para o fenótipo espesso, corroborando os dados de De Rouck ${ }^{8}$.

Outros estudos que reproduziram essa metodologia e que também classificaram os fenótipos em fino ou espesso, concluíram que o teste de transparência à sondagem é um método que, quando devidamente padronizado, é suficientemente reprodutível e confiável ${ }^{10,13-16}$. Dentre as principais características encontradas após a determinação dos fenótipos nessas pesquisas, foi visto que o fenótipo gengival fino apresenta tecido mole mais delgado com menor faixa de mucosa ceratinizada, papilas interdentais mais longas e estreitas. Já o fenótipo espesso foi caracterizado por apresentar maior faixa de mucosa ceratinizada com aspecto denso e fibrótico, bem como papilas mais curtas e largas ${ }^{10,13-16}$.

Ainda que os parâmetros contemplados pelos estudos sobre o fenótipo gengival sejam fidedignos, sabe-se que a arquitetura periodontal, por obedecer aos padrões fisiológicos do corpo humano, apresenta características geneticamente determinadas e dependem de fenômenos endógenos do organismo, como os que estão relacionados ao crescimento e envelhecimento, além das influências sofridas com perfil e posicionamento dos dentes 1,5-7,14,17.

Nesse sentido, esta pesquisa utilizou uma amostra relativamente jovem, insuficiente para superar o risco de vieses, tais como a atrição dentária fisiológica ou patológica, além de alterações no tecido gengival ocorridas ao avançar da idade, relacionadas ao adelgaçamento do epitélio e à diminuição da ceratinização $0^{6}$. Com relação à espessura gengival, alguns autores ${ }^{6,14}$ relataram que a gengiva é mais espessa na faixa etária mais jovem do que naquelas mais avançadas. Porém, segundo eles, pode haver influência de outros fatores de confusão na espessura gengival, como os aspectos genéticos, que precisam ser mais bem investigados ${ }^{6,14}$. Além disso, ainda são escassos os resultados na literatura que relacionam idade e demais parâmetros clínicos associados ao fenótipo gengival.

A fim de aprofundar a investigação sobre fatores intrínsecos de cada indivíduo, foi verificada a associação entre raça, sexo e exposição gengival com o fenótipo gengival. Como nas pesquisas de De Rouck et al. ${ }^{8}$ e Andand et al. ${ }^{10}$, também foi encontrada uma alta prevalência de fenótipos gengivais superiores finos para o sexo feminino e espessos para o sexo masculino. Segundo os autores, essa relação de gengivas espessas pode estar ligada às características fenotípicas relacionadas ao sexo, que determinam o arranjo de tecidos moles e o arcabouço ósseo humano, geralmente mais robustos no organismo masculino ${ }^{8,10}$. 
Foi vista, neste estudo, uma associação estatisticamente significativa entre indivíduos com fenótipo gengival superior espesso com exposição gengival ao sorrir maior que $2 \mathrm{~mm}$. Nesses casos, essa relação parece se dever à maior quantidade de tecido gengival existente no fenótipo espesso quando comparado ao fino, podendo ocasionar uma maior exposição de gengiva no ato de sorrir. Entretanto, não há estudos suficientes na literatura científica que estabeleçam, de forma concreta, essa relação.

Buscou-se ainda esclarecer a prevalência de fenótipos gengivais finos para a região anterior inferior e foi visto que esse achado está ligado ao fato de elementos dentários anteriores inferiores possuírem coroas mais delgadas e estrutura óssea disposta de forma mais compacta e afilada do que aquelas encontradas no arco superior. Esse aspecto acaba por aumentar o número de casos de fenótipos finos nessa região ${ }^{10,14}$.

Galgali, Gontiya ${ }^{18}$, que avaliaram radiograficamente e mediram as dimensões dentogengivais das superfícies vestibulares dos dentes anteriores, atribuíram à associação entre sexo e fenótipo gengival o fato de homens apresentarem maior quantidade de tecido gengival inserido e osso alveolar do que as mulheres. Essas características confirmaram relatos anteriores sobre o arranjo das estruturas anatômicas periodontais ${ }^{17,19}$. Além disso, não foram encontradas informações suficientes na literatura que justificassem a associação das dimensões do sorriso gengival com o fenótipo gengival, sugerindo que novos estudos devem ser realizados para analisar a relação entre esses dois parâmetros.

Em estudo observacional do tipo transversal, Egreja et al. ${ }^{20}$ verificaram a correlação do fenótipo gengival e as faixas de mucosa ceratinizada em caninos, incisivos laterais e incisivos centrais de 60 pacientes, encontrando uma ordem crescente de médias de tecido ceratinizado para canino, incisivos centrais e incisivos laterais, respectivamente, com médias de faixa de mucosa predominantemente maiores para indivíduos de fenótipo espesso. Outros autores avaliaram as médias desses grupos de dentes para ambos os arcos e também encontraram diferenças estatísticas, com médias de faixa de mucosa maiores para caninos e incisivos superiores do que para os inferiores, em respectiva ordem crescente ${ }^{17,20,21}$, assim como mostrado nesse estudo.

Os resultados deste estudo evidenciaram acentuada diferença tanto entre as médias de mucosa ceratinizada para caninos e incisivos superiores e inferiores quanto - ao serem comparadas entre si - as médias dos dois grupos de todos os dentes anteriores dos dois arcos. A informação de que dentes superiores têm quase o dobro da média de faixa de mucosa do que os inferiores e de que caninos têm menos tecido ceratinizado do que incisivos em ambos os arcos pode ser explicada pelos arranjos dos tecidos duros e moles característicos de cada tipo de dente, bem como pela localização de cada elemento dentário no arco ${ }^{10,17}$.

Esfahrood et al. ${ }^{21}$, em uma revisão sistemática, concluíram que, além da disposição do tecido ósseo vestibular, o formato e a posição das raízes de cada tipo de dente são cruciais na determinação da gengiva inserida e, consequentemente, da mucosa ceratinizada. Ademais, considerando-se que associações sexo/FG e MC/FG são estatisticamente significativas, quando confrontadas as informações entre MC e sexo, a MC é maior em dentes anteriores superiores nos homens, anteriormente associados à maior frequência de fenótipos espessos. Essa associação com maiores valores médios da faixa de tecido ceratinizado foi relatada anteriormente, confirmando os estreitos vínculos entre os parâmetros determinantes do fenótipo gengival já previstos por outros pesquisadore ${ }^{10,11}$.

Embora as características morfológicas da gengiva tenham fatores visuais determinantes, além daqueles naturalmente determinados, como a dimensão do processo alveolar e os eventos que ocorrem durante a erupção dentária ${ }^{22,23}$, outros aspectos devem ser levados em consideração, como a espessura da mucosa ceratinizada. Esse parâmetro clínico pode ser avaliado tanto por métodos invasivos quanto não invasivos, ambos igualmente eficazes e de fácil reprodutibilidade ${ }^{17,18}$. 
Um exemplo de método mais sutil e mais confiável emprega a introdução de um espaçador digital com cursor de silicone no tecido gengival, sob anestesia local. Essa técnica é eficaz e de fácil execução para avaliações em mucosa ceratinizada e permite a mensuração da espessura real do tecido gengival sem causar danos de qualquer natureza ao paciente ${ }^{17,19,24}$. 0 fato de a média da espessura gengival ser superior para tecidos moles superiores e ter correlação positiva com os valores médios da faixa de mucosa ceratinizada tem relação não somente com a maior faixa de tecido ceratinizado que ocorre em fenótipos espessos, mas também com o arranjo e a anatomia dos elementos dentários envolvidos, cujos formatos influenciam na disposição das estruturas periodontais circunjacentes ${ }^{3,17}$.

A diferença estatística de espessura gengival entre ambos os arcos examinados é justificada pela organização do arcabouço periodontal, dependendo não só de tecidos moles para cada tipo de maxilar como também dos tecidos ósseos: (a) osso alveolar espesso e gengiva espessa; (b) osso alveolar fino e gengiva espessa; (c) osso alveolar fino e gengiva fina; (d) osso fino e gengiva mediana ${ }^{17,20}$. Outros estudos empregaram metodologias semelhantes $18,20,24,25$ para mostrar uma diferença entre a espessura gengival superior e inferior, com maior prevalência de gengiva superior espessa que apresenta correlação positiva moderada com tecidos queratinizados, como visto no presente estudo.

0 reconhecimento das características de saúde periodontal de pacientes que procuram por tratamentos que envolvam estética e, sobretudo, restabelecimento funcional, é um dos aspectos primordiais na avaliação periodontal. Nessa perspectiva, a sondagem do periodonto está entre os mais relevantes métodos de escolha para uma avaliação periodontal e determinação do fenótipo de cada paciente ${ }^{8,10}$.

Sondas periodontais foram empregadas para realizar o teste de transparência à sondagem e estimar e diagnosticar os níveis de saúde periodontal em cada indivíduo para identificar seus respectivos fenótipos gengivais. Esses fatores podem modular o comportamento clínico das estruturas periodontais que determinam cada tipo de fenótipo. Contudo, não foi possível analisar as variações na arquitetura e na saúde periodontal com o volume gengival, dada a ausência de diferença estatística significativa e baixa correlação entre essas características encontradas neste estudo.

\section{CONCLUSÃO}

Verificou-se que a aferição da faixa de mucosa ceratinizada bem como a espessura gengival são parâmetros clínicos - relacionados aos tecidos moles circunjacentes em dentes anteriores superiores e inferiores - confiáveis e adequados à determinação do perfil de fenótipos gengivais em indivíduos periodontalmente saudáveis. Mais estudos que evidenciem a associação da exposição gengival ao sorrir e que determinem a influência direta da profundidade de sondagem na determinação do fenótipo gengival devem ser realizados.

\section{REFERÊNCIAS}

1. Cortellini P, Bissada NF. Mucogingival conditions in the natural dentition: narrative review, case definitions, and diagnostic considerations. J Periodontol. 2018 Jun;89(Suppl 1):S204-13. http://dx.doi.org/10.1002/JPER.16-0671. PMid:29926948.

2. Jepsen S, Caton JG, Albandar JM, Bissada NF, Bouchard P, Cortellini P, et al. Periodontal manifestations of systemic diseases and developmental and acquired conditions: Consensus report of workgroup 3 of the 2017 World Workshop on the Classification of Periodontal and Peri-Implant Diseases and Conditions. J Clin Periodontol. 2018 Jun;45(Suppl 20):S219-29. http://dx.doi.org/10.1111/jcpe.12951. PMid:29926500. 
3. Fischer KR, Künzlberger A, Donos N, Fickl S, Friedmann A. Gingival biotype revisited-novel classification and assessment tool. Clin Oral Investig. 2018 Jan;22(1):443-8. http://dx.doi.org/10.1007/s00784-017-2131-1. PMid:28551728.

4. Kahn S, Almeida RA, Dias AT, Rodrigues WJ, Barceleiro MO, Taba M Jr. Clinical considerations on the root coverage of gingival recessions in thin or thick biotype. Int J Periodontics Restorative Dent. 2016 May-Jun;36(3):409-15. http://dx.doi.org/10.11607/prd.2249. PMid:27100811.

5. Olsson $\mathrm{M}$, Lindhe J. Periodontal characteristics in individuals with varying form of upper central incisors. J Clin Periodontol. 1991 Jan;18(1):78-82. http://dx.doi.org/10.1111/j.1600051X.1991.tb01124.x. PMid:2045523.

6. Vandana KL, Savitha B. Thickness of gingiva in association with age, gender and dental arch location. J Clin Periodontol. 2005 Jul;32(7):828-30. http://dx.doi.org/10.1111/j.1600-051X.2005.00757.x. PMid:15966893.

7. Matarese G, Isola G, Ramaglia L, Dalessandri D, Lucchese A, Alibrandi A, et al. Periodontal biotype: characteristic, prevalence and dimensions related to dental malocclusion. Minerva Stomatol. 2016 Aug;65(4):231-8. PMid:27035270.

8. De Rouck T, Eghbali R, Collys K, De Bruyn H, Cosyn J. The gingival biotype revisited: transparency of the periodontal probe through the gingival margin as a method to discriminate thin from thick gingiva. J Clin Periodontol. 2009 May;36(5):428-33. http://dx.doi.org/10.1111/j.1600-051X.2009.01398.x. PMid:19419444.

9. Kan JY, Morimoto T, Rungcharassaeng K, Roe P, Smith DH. Gingival biotype assessment in the esthetic zone: visual versus direct measurement. Int J Periodontics Restorative Dent. 2010 Jun;30(3):237-43. PMid:20386780.

10. Anand V, Govila V, Gulati M. Correlation of gingival tissue biotypes with gender and tooth morphology: a randomized clinical study. Indian J Dent. 2012 Oct-Dec;3(4):190-5. http://dx.doi.org/10.1016/j.ijd.2012.05.006.

11. Shetty S, Bhat V. Prevalence of different gingival biotypes in individuals with varying forms of maxillary central incisors: a survey. J Dent Implant. 2013;3(2):116-21. http://dx.doi.org/10.4103/0974-6781.118888.

12. Lang NP, Bartold PM. Periodontal health. J Clin Periodontol. 2018 Jun;45(Suppl 20):S9-16. http://dx.doi.org/10.1111/jcpe.12936. PMid:29926485.

13. Cuny-Houchmand M, Renaudin S, Leroul M, Planche L, Guehennec LL, Soueidan A. Gingival biotype: the probe test utility. Open J Stomatol. 2013 Jan;3(2):123-7. http://dx.doi.org/10.4236/ojst.2013.32023.

14. Manjunath RG, Rana A, Sarkar A. Gingival biotype assessment in a healthy periodontium: transgingival probing method. J Clin Diagn Res. 2015 May;9(5):ZC66-9. http://dx.doi.org/10.7860/JCDR/2015/13759.5956. PMid:26155566.

15. Grover V, Bhardwaj A, Mohindra K, Malhotra R. Analysis of the gingival biotype based on the measurement of the dentopapillary complex. J Indian Soc Periodontol. 2014 Jan;18(1):43-7. http://dx.doi.org/10.4103/0972-124X.128199. PMid:24744543.

16. Fischer KR, Richter T, Kebschull M, Petersen N, Fickl S. On the relationship between gingival biotypes and gingival thickness in young Caucasians. Clin Oral Implants Res. 2015 Aug;26(8):865-9. http://dx.doi.org/10.1111/clr.12356. PMid:24580810.

17. Kolte R, Kolte A, Mahajan A. Assessment of gingival thickness with regards to age, gender and arch location. J Indian Soc Periodontol. 2014 Jul;18(4):478-81. http://dx.doi.org/10.4103/0972124X.138699. PMid:25210263.

18. Gontiya G, Galgali SR. Evaluation of an innovative radiographic technique - parallel profile radiography - to determine the dimensions of dentogingival unit. Indian J Dent Res. 2011 Mar-Apr;22(2):237-41. http://dx.doi.org/10.4103/0970-9290.84294. PMid:21891892. 
19. Alves PHM, Alves TCLP, Pegoraro TA, Costa YM, Bonfante EA, Almeida ALPF. Measurement properties of gingival biotype evaluation methods. Clin Implant Dent Relat Res. 2018 Jun;20(3):280-4. http://dx.doi.org/10.1111/cid.12583. PMid:29350855.

20. Egreja AM, Kahn S, Barceleiro M, Bittencourt S. Relationship between the width of the zone of keratinized tissue and thickness of gingival tissue in the anterior maxilla. Int J Periodontics Restorative Dent. 2012 Oct;32(5):573-9. PMid:22754905.

21. Esfahrood ZR, Kadkhodazadeh M, Talebi Ardakani MR. Gingival biotype: a review. Gen Dent. 2013 Jul;61(4):14-7. PMid:23823337.

22. Kahn S, Menezes CC, Imperial RC, Leite JS, Dias AT. Influência do biótipo periodontal na implantodontia e na ortodontia. Rev Bras Odontol. 2013;70(1):40-5.

23. Abraham S, Deepak KT, Ambili R, Preeja C, Archana V. Gingival biotype and its clinical significance - a review. Saudi J Dent Res. 2014 Jan;5(1):3-7. http://dx.doi.org/10.1016/j.ksujds.2013.06.003.

24. Frost NA, Mealey BL, Jones AA, Huynh-Ba G. Periodontal biotype: gingival thickness as it relates to probe visibility and buccal plate thickness. J Periodontol. 2015 Oct;86(10):1141-9. http://dx.doi.org/10.1902/jop.2015.140394. PMid:26110452.

25. Issrani R, Chavva S, Prabhu N, Keluskar V, Jirge V, Kumbujkar V, et al. Transgingival probing and ultrasonographic methods for determination of gingival thickness- a comparative study. Adv Hum Biol. 2013;3(3):43-51.

\section{CONFLITOS DE INTERESSE}

Os autores declaram não haver conflitos de interesse.

\section{*AUTOR PARA CORRESPONDÊNCIA}

Samuel Batista Borges, UFRN - Universidade Federal do Rio Grande do Norte, Departamento de Odontologia, Av. Senador Salgado Filho, 1787, Lagoa Nova, 059056-000 Natal - RN, Brasil, e-mail: sborges1990@gmail.com

Recebido: Fevereiro 12, 2019

Aprovado: Agosto 5, 2019 